\title{
LIPOSOMAL- AND CPG OLIGODEOXYNUCLEOTIDE-BASED VACCINE FORMULATIONS OFFER PROTECTION WITH REDUCED ADVERSE EFFECTS IN RAINBOW TROUT
}

\author{
Kasper Rømer Villumsen ${ }^{1 \S}$, Dennis Christensen², Erling Olaf Koppang ${ }^{3}$ \& Anders Miki \\ Bojesen $^{1}$ \\ ${ }^{1}$ Institute of Veterinary Disease Biology, University of Copenhagen, Frederiksberg, Denmark \\ ${ }^{2}$ State Serum Institute, Copenhagen, Denmark \\ ${ }^{3}$ School of Veterinary Medicine, Norwegian University of Life Sciences, Oslo, Norway
}

\begin{abstract}
Mass-scale, intraperitoneal administration of vaccines containing mineral oil-adjuvants, has been common practice in salmonid aquaculture since the early 1990's. Introduced as a response to the threat of disruptive outbreaks of aquatic pathogens such as Aeromonas salmonicida subspecies salmonicida, the causative agent of furunculosis, such formulations have increased the survival rates and profitability of the farming of these species.

Nevertheless, well-documented local adverse effects, including granulomatous inflammation with melanisation and adhesions of the peritoneal cavity, in addition to systemic effects, have also been observed. Here, aiming at exploring alternatives to mineral oil-adjuvanted furunculosis injection vaccines, three different vaccine formulations were designed and administered to rainbow trout (Onchorhynchus mykiss) fry. All three formulations were based on whole-cell A. salmonicida subspecies salmonicida bacterin, formulated with either Freund's incomplete adjuvant (FIA), dimethyldioctadecylammonium/trehalose 6,6'dibehenate liposomes (CAF01), or CpG oligodeoxynucleotides. Non-vaccinated, TBSinjected and bacterin-injected groups were included as control groups. Following vaccination, fish were sampled in order to monitor the occurrence of adverse effects, as well as the development of the immunological reaction to each vaccine formulation. Finally, a waterborne challenge with $A$. salmonicida was performed to assess the protection obtained in each experimental group. Significant differences were observed with regards to the occurrence of adverse effects, both in individual scoring of adhesions and pigmentation, as well as in the combining Speilberg score, demonstrating a general reduction in adverse effects in the CpG- and CAF01-group, compared to individuals injected with the FIA-formulation. When analyzing serum samples, significantly elevated antibody titers were seen in all three experimental formulations when compared to non-vaccinated controls, and finally a significant, uniform protective effect was seen in all three vaccine groups.

While this is still an ongoing study, the combination of protection from infection along with the reduction in adverse effects achieved by the use of liposomal and nucleotide adjuvants in this study, is promising with respect to alternative, low-toxicity adjuvants in aquaculture.
\end{abstract}

\section{KEYWORDS:}

Vaccines, adjuvants, adverse effects, rainbow trout, salmonids.

${ }^{\S}$ Corresponding author. Tel.: +45 35336763

E-mail address: krv@sund.ku.dk 